\title{
Voltaire précurseur d'un nouveau genre dramatique
}

\section{Dr. Adli Abdel Raouf ${ }^{(*)}$}

Notons qu'à partir du premier tiers du XVIIIe siècle le mot romantisme commence à faire figure d'une modeste apparition auprès du nom de Voltaire. René Pommeau écrit, dans son Voltaire que "certaines parties de l'œuvre de Voltaire annonce le romantisme ${ }^{\mathbf{1}}$. Et Il ajoute un peu plus loin que "toutes les frénésies du drame romantique sont déjà dans les tragédies de Voltaire"2. Aussi, Raymond Naves affirme que "Voltaire critique et pote, mieux qu'aucun critique contemporain conduit à Chénier et à Lamartine, c'est à dire à ces romantiques tempérée qui conservent la noblesse et le goût dans les élans de leur inspiration"3. Nous nous demandons maintenant ce que le théâtre de Voltaire avait du romantisme et dans quelle mesure nous pouvons le qualifier de théâtre romantique. Nous allons aborder cette question en essayant de relever les éléments du romantisme qui se trouvent dans son théâtre.

Voltaire s'efforce de maintenir le pathétique dans ses tragédies. La tragédie pathétique lui paraît nécessaire. Il fait naitre l'émotion et touche la sensibilité ${ }^{4}$. Dans Zaïre, la peinture

\section{(*) Maître de Conférences}

$\left.{ }^{1}\right)$ René Pommeau Voltaire. p. 53

2) Ibid. P. 58

3) Cf. Voltaire, l'Homme et l'Euvre, P; 107)

$\left.{ }^{4}\right)$ Les Maitres de la sensibilité au XVIIIe Siècle, T I. P. 237) 
d'une jeune fille partagée entre son amour pour son amant Orosmane et son devoir à l'égard de son pères et de son frère est également touchante. Il déclare lui-même à propos de cette pièce dans Le Mercure de France le 25 août 1732: " Zaïre est la première pièce dans laquelle, j'ai osé m'abandonner à toute la sensibilité de mon cœur"1 ${ }^{1}$. Cette déclaration pose Voltaire, à notre avis, dans l'atmosphère de la sensibilité préromantique.

Mais comment Voltaire introduit-il le pathétique dans ses tragédies? D'abord en choisissant des sujets pleins de grands intérêts et qui ne roulent pas seulement sur un mariage, d'où ses tragédies historiques et politiques, ensuite en traitant l'amour avec toute la violence qu'il comporte, enfin en plaçant, grâce à une suite rapide d'événements un attendus pour les personnages et même pour les spectateurs, ses héros et ses héroïnes dans une séries de situations neuves, fortes, à la fois surprenantes et naturelles. ${ }^{2}$

Voltaire cherche les situations bouleversantes. Il répète toujours l'imbroglio des enfants volés ou perdus qui sont au théâtre des enfants retrouvés ${ }^{3}$; dans Zaïre, dans Mérope etc...

Le poignard, l'inceste, le suicide, l'incendie, le poison dont son théâtre est rempli, et nous l'avons trop remarqué, ne jouent-ils pas un rôle important dans l'armure du mélodrame? Certes oui, et le mélodrame mène à son tour au drame romantique.

\footnotetext{
1) Correspondance, TI , P. 323

${ }^{2}$ ) Les tragédies et les théories dramatiques de Voltaire, P. 433

$\left.{ }^{3}\right)$ René Pommeau: Voltaire P. 58
} 
René Pommeau affirme que le sujet de Sémiramis est plus romantique que celui d'Hernani car dans Hernani, il n'y a pas de fantôme, mais dans Sémiramis, il y a un fantôme, celui du père assassiné en provenance d'Hamlet...Dans Olympie, l'héroïne se jette sous les yeux des spectateurs dans un braiser, un vrai braiser qui, au cours d'une représentation, mit le feu au théâtre de Voltaire'.

Nous pensons que les tragédies de Voltaire sont des mélodrames. Aussi, René Pommeau pense-t- il qu'après son Edipe " Voltaire ne fera plus sous le nom des tragédies que de pathétiques mélodrames où s'agite une humanité qui ne connaît plus Dieu"2.

Par conséquent, Voltaire use et abuse des quiproquos, des méprises, des incognitos, des surprises, des revirements, des coups de théâtre, des reconnaissances retardées et provoquées arbitrairement. Ces moyens dont il abuse seront plus tard moyens de mélodrame ou de vaudeville. Emile Faguet affirme à son tour que les tragédies de Voltaire "sont tellement des mélodrames qu'elles commencent déjà à être des vaudevilles. On sait qu'entre le mélodrame moderne et le Vaudeville, il n'y a aucune différence de fond. L'un est fondé sur une ou plusieurs méprises, l'autre sur un ou plusieurs quiproquos. Et la méprise n'est qu'un quiproquo triste et le quiproquo n'est qu'une méprise gaie, et les personnages du mélodrame doivent se prêter complaisamment à la méprise et les personnages du Vaudeville s'ajuster de leur mieux au

\footnotetext{
1) Ibid. P.59.

2) La Religion de Voltaire P. 91
} 
quiproquo. Les tragédies de Voltaire ont déjà nettement ce caractère ${ }^{1}$.

Voltaire incline la tragédie vers le mélodrame également par la recherche du pathétique et par la recherche des tableaux attendrissants ou terribles. Les grandes scènes de son théâtre: Mérope levant la hache sur son fils, Orosmane poignardant Zaïre, Jocaste se suicidant devant nos yeux, l'exposition du cadavre de César sous une robe sanglante aux yeux des spectateurs, le corps de Polyphonte couvert une sanglante sont "des scènes de pure pathétique presque sans contenu psychologique et d'un pathétique bien théâtral qui ne peut se passer des moyens scéniques d'exécution et ne donne tout son effet qu'aux yeux. Du Belloy, Lemierre, Durcis n'ajoutent à l'action de Voltaire que des hardiesses particulières: ils marchent quand Voltaire s'est arrêté. On n'est plus là, mais dans le sens qu'il indiqué. Il est le plus grand nom représentatif par lequel peut s'éclairer le passage de la tragédie classique au drame romantique, d'Athalie à Hernani" ${ }^{2}$

Il remplace la psychologie tragique de Racine qui fait longueur par des incidents car toutes les tragédies françaises lui paraissent trop longues et remplace le détail psychologique de Racine par un détail, matériel, c'est-à-dire qu'il a créé le mélodrame. $^{3}$

Dans La Mort de César, Voltaire donne un nouvel exemple de sa conception sentimentale de la tragédie et du

\footnotetext{
1) Cf . Le XVIIIe siècle, Etudes littéraires, P. 265

$\left.{ }^{2}\right)$ G. Lanson: Voltaire P. 105

${ }^{3}$ ) Emile Faguet: XVIIIe siècle, Etudes littéraires, P. P. 260-261
} 
tragique où les émotions plutôt fortes que douces, suscitées par des problèmes familiaux juxtaposés à une idéologie de propagande, rattachant la pièce à une dramaturgie qui ne s'épanouira que plus tard, c'est-à-dire le mélodrame'

Certaines de ses pièces sont des avant-coureurs du drame romantique du XIXe siècle comme Zaïre, Tancrède, Mérope, La Mort de César etc.....Les tragédies de Voltaire préparent le drame romantique par le pathétique violent, par la fièvre de l'action, par le souci du décor, de prédication sociale, politique et religieuse.

C'est ainsi que le drame romantique est plus venu plus tard plutôt de théâtre de Voltaire que de celui de Shakespeare et de celui de Diderot. Et nous nous appuyons d'Emile Faguet lorsqu'il dit que "les drames romantiques de 1830 sont des tragédies de Voltaire enluminées de métaphores ${ }^{2}$.

Aussi, le drame historique est-il issu des tragédies de Voltaire plutôt que des théories de Diderot. Le Drame en France au XVIIIe siècle, P. 98 Aussi le drame sérieux est-il préparé par quelques drames sérieuses de Voltaire et par la comédie larmoyante de Nivelle de la Chaussée, mais il ne date pas vraiment que du Fils Naturel de Diderot, publiée en 1757, mais jouée une seule fois seulement en $1771^{3}$.

C'est ainsi que Voltaire annonce une transformation prochaine de la tragédie classique en drame. Mais ce genre plus large et plus varié ne s'épanouit qu'à l'époque romantique. Et

\footnotetext{
1) André Rousseau: Voltaire, La Mort de César, P.19

$\left.{ }^{2}\right)$ Emile Faguet: XVIIIe siècle, Etudes littéraires, P. 272

${ }^{3}$ ) La dramaturgie de Beaumarchais P. 19
} 
nous pensons avec Gustave Lanson que "la tragédie voltairienne aurait donc pour formule une carcasse de Vaudeville ou de mélodrame construite pour donner lieu à l'effusion lyrique. Mais justement, ce sera la formule romantique de Victor Hugo"1

Si les tragédies de Voltaire annonçaient le didactisme et l'idéologie du drame, ses comédies le préparent ainsi par l'attendrissement du ton, l'actualité de l'intrique et la moralité de l'intention, Voltaire avait pressenti tout le parti pris à tirer d'une habile juxtaposition du comique et du sérieux. Il loue les attraits "d'un genre mixte apte à concilier le plaisir du sourire et le bonheur de l'émotion sans tomber dans l'erreur d'une comédie sans comique" ${ }^{2}$.

Remarquons que Voltaire mélange quelquefois le tragique et le comique, c'est-à-dire, il incline la tragédie vers le naturel de la comédie et la comédie à son tour vers le pathétique de la tragédie. La vraie nature de la comédie, chez lui, est un mélange d'un pathétique et d'un comique également discret comme dans la vie courante. Dans la Préface de Nanine ou le Préjugé Vaincu, il nous paraît que Voltaire n'admet pas la comédie larmoyante qui lui semble "un genre vicieux et très désagréable ....Ce serait une espèce batarde, un monstre né de l'impuissance de faire une comédie et tragédie véritable. ... Il blâme surtout les intriques romanesques et forcées dans ce genre de comédie où l'on veut attendrir les spectateurs et qu'on

\footnotetext{
$\left.{ }^{1}\right)$ Cf. esquisse d'une histoire de la tragédie française , P. 149

${ }^{2}$ ) Michel Lioure : Le Drame de Diderot à Ionesco, P. 14
} 
appelle par dérision, comédie larmoyante"1, Mais, il écrit dans son article Art Dramatique, Comédie, "qu'on ne travaille dans le goût de la comédie larmoyante que parce que ce genre est plus aisé, mais cette facilité le dégrade: en un mot, les Français ne surent rire."2

Il nous paraît que le rire et l'émotion coexistent et se succèdent dans ses comédies. C'est ainsi que Voltaire admet un genre intermédiaire entre la tragédie et la comédie; c'est-àdire le mélodrame, dans La préface de l'Enfant Prodigue, Voltaire constate que, puisque dans "ce siècle raisonneur", on est porté au sentiment, il bon d'introduire dans la comédie "un mélange sérieux et de plaisanteries de comique et de touchant." ${ }^{3}$ Il ne s'agit pas que "toute comédie doive avoir des scènes de bouffonneries et des scènes attendrissantes. Il y a beaucoup de bonnes pièces où il ne règne que de la gaieté; d'autres toutes sérieuses, d'autres mélangées, d'autres où l'attendrissement va jusqu'aux larmes" Ibid. C'est ainsi qu'il ne faut exclure aucun genre " tous les genres sont bons, hors les genres ennuyeux"4.

La comédie que Voltaire conçoit n'est pas éloignée de ce que Dumas Fils et Augier réaliseront après 1850. Sa comédie, neuve à l'époque, est indépendante des modèles préconçus. Il s'en dégage que la comédie est un genre moyen qui, entre le rire mécanique et l'émotion qu'elle exclut tous les deux, établit de la manière la plus large possible un rapport entre le

\footnotetext{
${ }^{1}$ ) Cf. Voltaire ; œuvres complètes, T. IV. P. 6

2) Op. Cit. P. 116

$\left.{ }^{3}\right)$ Voltaire: (Euvres complètes, T.II. P. 443

$\left.{ }^{4}\right)$ Ibid. P.445
} 
spectateur et le monde où il vit. Les mœurs contemporaines, les formes de la sensibilité contemporaines, les problèmes contemporains, s'y trouvent donc évoqués comme dans la comédie des mœurs ${ }^{1}$.

Le Café ou l'Ecossaise introduit en France la tradition d'un théâtre d'atmosphère anglaise dont Chatterton de Vigny doit être le chef d'œuvre et l'on y trouve plus d'un trait du drame de Vigny: "le contraste entre une ambiance générale de cordialité un peu vulgaire et la souffrance de certains êtres blessés dans leur délicatesse et la tentation du suicide qui hante ces derniers."

Enfin, nous remarquons que la comédie de Voltaire se situe à mi-chemin entre la tragédie et la comédie. Et Voltaire travaille de son mieux pour renouveler la cadre traditionnel de la comédie. Il fait la théorie d'un genre mixte tout nouveau où les genres sont mêlés; c'est-à-dire le drame sérieux

Avec lui, la tragédie et la comédie tendent à se confondre, à se joindre en un genre intermédiaire, ce genre n'est autre que le mélodrame et le mélodrame a préparé la voie au drame romantique de Victor Hugo et de Dumas ${ }^{3}$.

Mais par quel côté le mélodrame annonce-t-il le drame romantique? On pourrait dire qu'il l'annonce par le goût de la complication romanesque, c'est-à-dire par des incidents, par des coups de théâtre, par des méprises, par des quiproquos, par des reconnaissances etc.... et nous avons remarqué que le

\footnotetext{
1) Pierre Voltz: La Comédie, Col. U. Armand-Collin, Paris, 1964, PP. 107-108

$\left.{ }^{2}\right)$ Le Café ou l'Ecossaise, Notice, P. 1399

${ }^{3}$ ) Le Drame en France au XVIIIe Siècle, P.552
} 
théâtre de Voltaire est fertile de ces moyens. Le mélodrame annonce aussi le drame romantique par le mélange du tragique et du comique. Donc, le drame romantique doit beaucoup plus à Voltaire qu'à Diderot, c'est ainsi, que l'on pourra dire que si J.J. Rousseau est le précurseur du romantisme français au XIXe siècle. Voltaire, à son tour, est l'auteur qui a préparé la voie à la création du drame romantique.

\section{Bibliographie}

- Emile Faguet Le XVIIIe siècle, Etudes littéraires, Paris, Société Française d'imprimerie et de librairie 1901

- Pommeau René Voltaire, Paris, Ecrivains de Toujours/Seuil, 1983

- Pommeau René. La Religion de Voltaire Paris, Nizet, 1974

- $\quad$ G. Lanson: Voltaire, Paris, Hachette, 1956.

- Lanson G; Esquisse d'une histoire de la tragédie française, Paris, Champion, 1957

- Michel Lioure : Le Drame de Diderot à Ionesco, Paris, Armand-Collin, 1963

- Pierre Voltz: La Comédie, Col. U. Armand-Collin, Paris, 1964, PP. 107-108

- Rousseau André: Voltaire, La Mort de César, Paris, Société d'édition et d'enseignement supérieur, 1964

- Navet Raymond; Voltaire, l'Homme et l'Euvre, Col. Connaissance des Lettres, Paris, Hatier, 1958

- Trachard, Pierre Les Maitres de la sensibilité française au XVIIIe Siècle, T. I , Paris, Boivin; 1931 
- Lion Henri; Les tragédies et les théories dramatiques de Voltaire, Genève, Slatkints, 197à

- Gaiffe Félix: Le Drame en France au XVIIIe siècle, Paris, Armand-Collin, 1971

- Voltz Pierre, La Comédie, COL, U, Paris, Armand-Collin, 1970

- Schérer Jacques La dramaturgie Classique en France , Paris, Nizet, 1954

- Schérer Jacques La dramaturgie de Beaumarchais, Paris, Nizet, 1980

- Voltaire: Correspondance, 1709-1738 TI , Paris, Bibliothèque de la Pléiade, Gallimard, 1963

- Voltaire ; œuvres complètes,, Paris, Garnier-Frères, S. D:, 43, Volumes 